\title{
Desalination process of penicillin wastewater and recovery of sodium sulfate
}

\author{
Yangkang Shao ${ }^{1, \text { a }}$, Kui Chen ${ }^{1, b}$, Jiawen Zhu ${ }^{1}$, Xiping Ying ${ }^{2}$ and Shengwu \\ Yang $^{2}$ \\ ${ }^{1}$ Chemical Engineering Research Center, East China University of Science \& Technology, Shanghai \\ 200237, China \\ ${ }^{2}$ Hulunbuir Northern Pharmaceutical Co. Ltd., Yakeshi 022150, China \\ a411979312@qq.com, bchenkui@ecust.edu.cn
}

Keywords: desalination; penicillin wastewater; sodium sulfate; solubility; antisolvent crystallization Abstract. In this study, a desalination procedure has been developed in penicillin G-processing wastewater biological treatment. The procedure is focused on removal of part of organics and the majority of sodium sulfate to decrease the difficulties in subsequent biological treatment. The effects of activated carbon, sodium hydroxide on the process have been carefully measured to enhance the COD removal rate. Based on solubility of sodium sulfate in a methanol-water system and the practical research, antisolvent crystallization process was applied to recover sodium sulfate with an optimal solvent volume ratio. Yield and purity of the refined crystals have finally reached $78.28 \mathrm{wt} \%$ and $97.56 \%$, respectively.

\section{Introduction}

Penicillin G-processing streams usually have complex components[1], containing high concentrated salts and organic compounds. The presence of sulfate radicals[2] has brought a great difficulty to the biological treatment of penicillin wastewater[3,4]. It has been a challenge to separate organics and recycle sodium sulfate with high efficiency and high economy.

We have recently developed an efficient approach to sodium sulfate separation from penicillin G-processing wastewater based on antisolvent crystallization using methanol. In our consideration, protein removal and desalination are both necessary for getting an ideal treatment effect of wastewater. Thus, to begin with, part of the organics can be removed by activated carbon adsorption and chemical precipitation. Alkaline proteins and derivatives from penicillin degradation can be easily precipitated by controlling the $\mathrm{pH}$ values. Subsequently, the wastewater is evaporated to obtain concentrated sodium sulfate solution. Sodium sulfate can thus be separated from the concentrated liquor by antisolvent crystallization[5], and recrystallized to meet the high quality requirement. The crystallization mother liquor, in which sulfate concentration have been reduced by one magnitude, are finally sent to the biological treatment process. These operations are focused on the removal of organics and sodium sulfate to enhance the biological treatment effect.

\section{Materials and methods}

Wastewater and materials. The practical penicillin G-processing wastewater was obtained from HulunBuir Northern Pharmaceutical Co., Ltd (Yakeshi, China). It was mainly generated from fermentation broth and refined process, and was characterized for physical, chemical and biological properties according to standard methods. The Bradford method was applied to measure the content of protein by using Spectrophotometer, while potassium dichromate method was used to the measurement of chemical oxygen demand (COD). All reagents involved were of analytical reagent grade. The determined main parameters of penicillin processing wastewater were listed in Table 1. 
Table 1. The components of the raw penicillin G-processing wastewater.

\begin{tabular}{ccc}
\hline Indicator & Concentration range & Unit \\
\hline $\mathrm{COD}_{\mathrm{cr}}$ & $14,000-23,500$ & {$\left[\mathrm{mg} \cdot \mathrm{L}^{-1}\right]$} \\
Suspended solid & $1,850-2,200$ & {$\left[\mathrm{mg} \cdot \mathrm{L}^{-1}\right]$} \\
$\mathrm{SO}_{4}{ }^{2-}$ & $1,250-1,650$ & {$\left[\mathrm{mg} \cdot \mathrm{L}^{-1}\right]$} \\
pH & $3.5-5.4$ & - \\
$\mathrm{BOD}$ & $1,400-1,750$ & {$\left[\mathrm{mg} \cdot \mathrm{L}^{-1}\right]$} \\
\hline
\end{tabular}

Desalination process. Fig. 1 summarized the procedure for producing crystalline sodium sulfate from Penicillin processing wastewater. The whole desalination procedure also can be divided into three different portions as follows:

Pretreatment: The raw wastewater was centrifuged by a high-speed centrifuge and the insoluble solids were decanted. $100 \mathrm{ml}$ filtrate was taken from the resultant solution and added into a $250 \mathrm{~mL}$ conical glass flask to perform an adsorption operation. A certain amount of activated carbon (AC) was added and the flask was maintained at a required constant temperature with agitation at $200 \mathrm{rpm}$ for 1 $\mathrm{h}$. Furthermore, a certain amount of solid sodium hydroxide was added into $50 \mathrm{~mL}$ solution and the solution was agitated to dissolve alkali completely. Then agitation was stopped and the mixture was kept for $0.5 \mathrm{~h}$ to enable any solid matter to settle. The precipitation of insoluble materials were removed by filtration, dried and weighted.

Preparation of crystalline sodium sulfate: Sodium sulfate solution was concentrated by evaporation. Methanol addition was achieved by using a peristaltic pump. An electronic balance was used for recording the mass of methanol added to the solution to reach a setting volume ratio between methanol and solution. Agitation of the solution was provided at a value of $200 \mathrm{rpm}$ for $30 \mathrm{~min}$. The crude crystals of anhydrous sodium sulfate was crystallized from methanol aqueous solution by adding a very small amount of seed crystals of sodium sulfate.

Recrystallization: The crude crystals obtained above were recrystallized from aqueous solution by cooling crystallization. The crude crystals were dissolved in $100 \mathrm{~mL}$ water by heating. After cooling to $0{ }^{\circ} \mathrm{C}$, a very small amount of seeds was added to induce nucleation. The formed crystals were separated from the mother liquor by filtration, and then dried in the oven at $110^{\circ} \mathrm{C}$ for about $2 \mathrm{~h}$. 


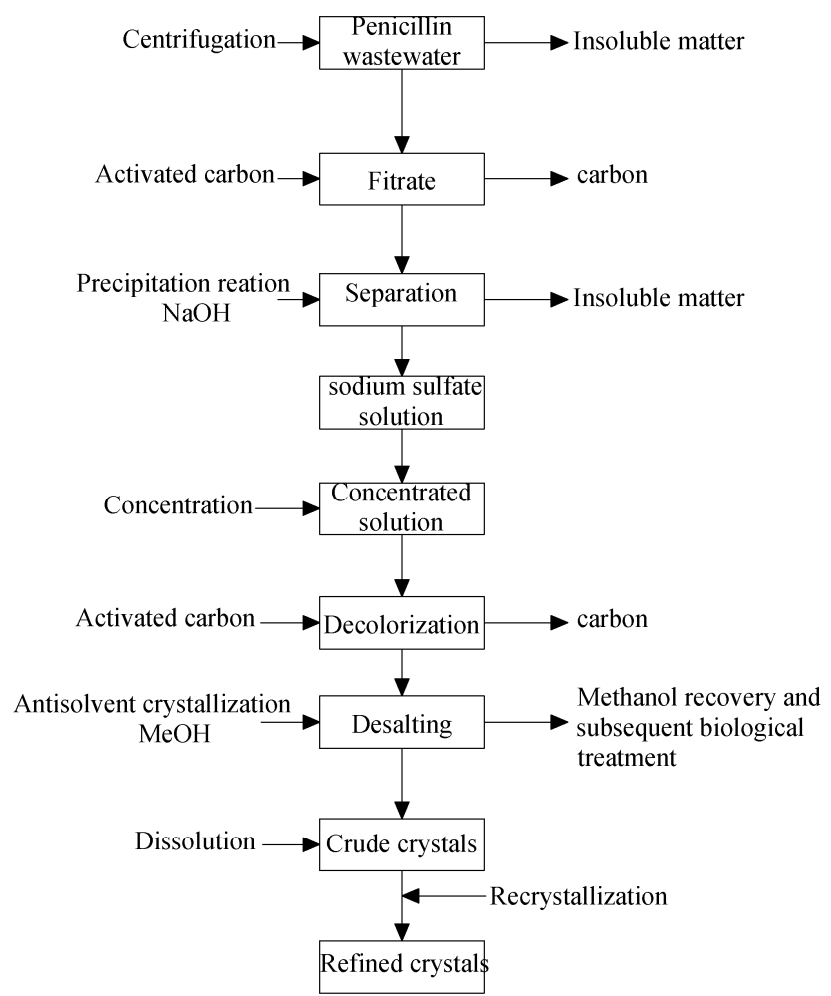

Fig. 1 Procedure for recovering crystalline sodium sulfate from Penicillin wastewater.

\section{Results and discussion}

Adsorption by activated carbon. Activated carbon (AC) has been recognized as one of the oldest and widely used adsorbent for removal of organic and inorganic pollutants. Fig. 2 shows the effect of AC addition on removal of the pigments. The absorbance of the resultant solution was measured by SPECORD ${ }^{2} 50 P L U S$. It shows that the removal rate of pigment rises with increasing of AC, while the adsorption efficiency has continued to decrease. This conclusion can be proved by Table 2 .

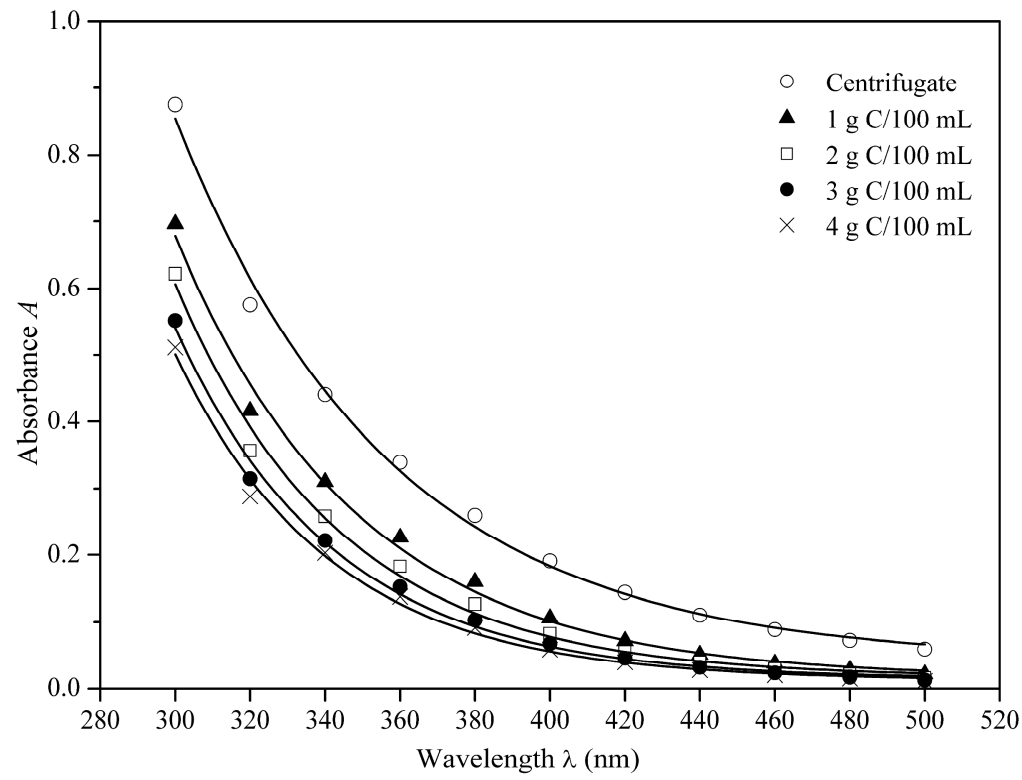

Fig. 2 The absorbance curves of solutions with different dosages of activated carbon. 
Table 2. The adsorption effect of AC on removal rates of protein and COD.

\begin{tabular}{ccccc}
\hline Activated carbon [g/L] & 10.0 & 20.0 & 30.0 & 40.0 \\
\hline Remove rate of protein [\%] & 68.33 & 77.90 & 83.21 & 86.72 \\
Remove rate of $\mathrm{COD}_{\mathrm{cr}}[\%]$ & 14.11 & 14.49 & 20.39 & 24.73 \\
\hline
\end{tabular}

Organics precipitation. Penicillin wastewater contains a large amount of acidic protein and penicillin and derivatives from penicillin degradation. The initial $\mathrm{pH}$ value of raw wastewater was about 5.67 and part of the organics would be precipitated by adding alkali to an appropriate $\mathrm{pH}$ range. The effect of sodium hydroxide addition on precipitation reaction was shown in Table 3 . The results indicate that $\mathrm{pH}$ and the mass of precipitation both increase with increasing sodium hydroxide addition.

Table 3. The effect of sodium hydroxide addition on $\mathrm{pH}$ and mass of precipitation.

\begin{tabular}{cccccccc}
\hline$m_{\mathrm{NaOH}}\left[\mathrm{g} \cdot \mathrm{L}^{-1}\right]$ & 0 & 2 & 4 & 6.0 & 8.0 & 12.0 & 16.0 \\
\hline $\mathrm{pH}$ & 5.67 & 6.23 & 6.76 & 7.89 & 8.63 & 9.27 & 9.75 \\
$m\left[\mathrm{~g} \cdot \mathrm{L}^{-1}\right]$ & 0 & 0.9361 & 3.8564 & 5.4305 & 5.4528 & 5.5967 & 5.8760 \\
\hline
\end{tabular}

Antisolvent crystallization. To investigate the effect of the initial content of methanol, solubility of sodium sulfate in methanol aqueous solutions was determined in a temperature range $\left(10\right.$ to $\left.50{ }^{\circ} \mathrm{C}\right)$ by means of the residue solid technique. In this technique, solubility was measured by equilibrating anhydrous sodium sulfate crystals and the mixed solvents at constant temperature. On the other hand, solubility data of sodium sulfate in methanol-water systems in the previous literature[6,7] show that there are some discrepancies among themselves. As shown in Fig. 3, for each temperature point, the solubility deceases evidently with increment in solvent mass ratio $(x)$. However, there is only a slight change of solubility after the solvent mass ratio, $x \geq 1$. Another conclusion is that the solubility is greatly affected by temperature, but it varies slightly after $t>40{ }^{\circ} \mathrm{C}$.

A power exponent type function was found to satisfactorily correlate the variation of solubility with the mass ratio of methanol to water:

$$
S=a /\left(b+x^{c}\right)
$$

where $a, b$ and $c$ are the fitting parameters; $x$ is the solvent mass ratio between methanol and water, $g$. $\mathrm{MeOH} / \mathrm{g} . \mathrm{H}_{2} \mathrm{O}$. The characteristic parameters were given in Table 4 . The relevant index $\mathrm{R}^{2}$ indicates that this expression describes well with the experimental data.

According to solubility measurement data, the process conditions of antisolvent crystallization will be at solvent mass ratio, $x=1$, when converted into volume solvent ratio is about 1.25 . Besides, the crystallization temperature should be as low as possible to reduce the volatilization of methanol. The practical experimental results were shown in Table 5. Though the yield of sodium sulfate increases with the raising of solvent volume ratio, however, purity of crude crystals continues to decline as some soluble materials were precipitated from solution because of the addition of methanol. In addition, due to the high operating cost in industrial scale, it is impossible to apply a relative high solvent volume ratio. Therefore, the selective optimal volume ratio in this study is 1 .

The crude crystals obtained above were recrystallized by cooling crystallization for further purification. After recrystallization, purity of the refined crystals obtained has been greatly improved with purity of $97.56 w t \%$, and reached the class II qualified purity of industrial anhydrous sodium sulfate standard (GB6009-92) requirements. 


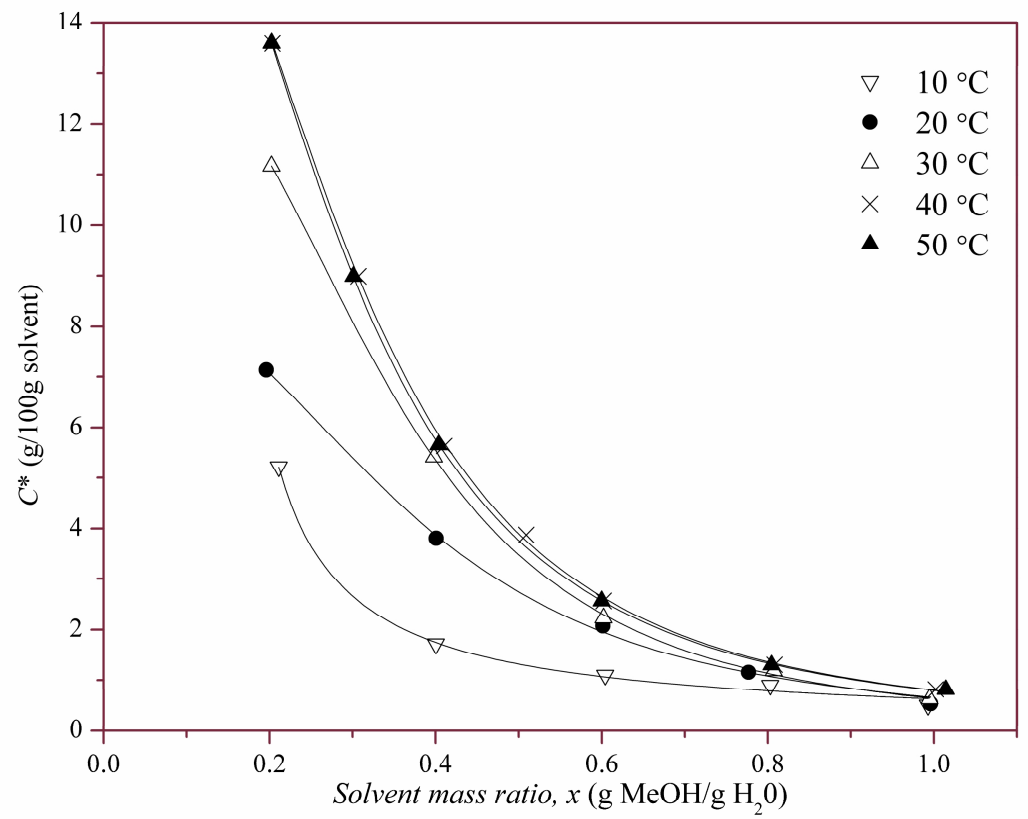

Fig. 3 Solubility of sodium sulfate in methanol-water solutions with solvent mass ratio at different temperatures and fitting curves by Eq. 1 .

Table 4. Fitting parameters a, b and $\mathrm{c}$ for solubility of sodium sulfate in methanol aqueous solutions in the temperature range from 10 to $50^{\circ} \mathrm{C}$.

\begin{tabular}{ccccc}
\hline$t\left[{ }^{\circ} \mathrm{C}\right]$ & $a$ & $b$ & $c$ & $\mathrm{R}^{2}$ \\
\hline 10 & 0.5076 & -0.1849 & 0.8128 & 0.9956 \\
20 & 0.7030 & 0.0823 & 2.5214 & 0.9977 \\
30 & 0.6584 & 0.0480 & 2.8234 & 0.9997 \\
40 & 0.8323 & 0.0443 & 2.5634 & 0.9992 \\
50 & 0.8325 & 0.0409 & 2.4670 & 0.9999 \\
\hline
\end{tabular}

Table 5. The yield and purity of crude crystals of sodium sulfate after antisolvent crystallization at different solvent volume ratios.

\begin{tabular}{ccccc}
\hline Solvent volume ratio & 0.5 & 1 & 2 & 3 \\
\hline Yield [wt \%] & 73.28 & 85.91 & 90.07 & 91.58 \\
Purity [wt \%] & 83.61 & 62.71 & 56.65 & 52.29 \\
\hline
\end{tabular}

\section{Conclusions}

A new desalination process of penicillin wastewater has been developed by using activated carbon adsorption and antisolvent crystallization. The amount of activated carbon and sodium hydroxide has been investigated by analysis of properties of penicillin wastewater. The solubility of sodium sulfate has been measured as a function of composition, indicating that the equilibrium sodium sulfate concentration in methanol aqueous solution varies greatly with temperature and solvent composition, with a relatively optimal value observed at solvent volume ratio $=1$ and corresponding to $85.9 \%$ sodium sulfate removal in practical experiments. These results provide the basis for identifying the optimal conditions for efficient and quick sodium sulfate removal from penicillin wastewater by antisolvent crystallization. Yield and purity of the refined crystals have reached $78.28 \mathrm{wt} \%$ and $97.56 \%$, respectively. The difficulties in treatment of penicillin wastewater and the volume of wastewater will both decrease greatly. 


\section{References}

[1] Z.D. Chang, X.F. Wei, H.Z. Liu, J.Y. Chen. Simulated foam separation of butyl acetate from wastewater discharged by solvent extraction operation in penicillin production. Separ. Sci. Technol. 2002, 37(4), 981-991.

[2] Yuan-feng Qi, Sheng-bing He, Su-qing Wu, Bi-bo Dai, Chang-hua Hu. Utilization of micro-electrolysis, up-flow anaerobic sludge bed, anoxic/oxic-activated sludge process, and biological aerated filter in penicillin G wastewater treatment. Desalination and Water Treatment, 2015, 55(6), 1480-1487.

[3] J.P. Maree, W. F.Strydom. Biological sulphate removal from industrial effluent in an upflow packed bed reactor. Water. Res. 1987,21(2), 141-146.

[4] P. Mullai, S. Arulselvi, Huu-Hao Ngo, P.L. Sabarathinam. Experiments and ANFIS modelling for the biodegradation of penicillin-G wastewater using anaerobic hybrid reactor. Bioresource Technol. 2011, 102(9), 5492-5497.

[5] J. Hash, O.C. Okorafor. Crystal size distribution (CSD) of batch salting-out crystallization process for sodium sulfate. Chem. Eng. Process. Process Intensif. 2008,47(4), 622-632.

[6] O.C. Okorafor. Solubility and density isotherms for the sodium sulfate-water-methanol system. $J$. Chem. Eng. Data. 1999, 44(3), 488-490.

[7] W. Fleischmann, A. Mersmann. Solubility, density, and viscosity for sodium sulfate-methanol-water systems at 40 degree. C. J. Chem. Eng. Data. 1984, 29(4), 452-456. 